\title{
B-Zell-Lymphom: Verzicht auf Bestrahlung ist keine gute Idee
}

\begin{abstract}
Ob bei einem diffusen großzelligen B-Zell-Lymphom (DLBCL) eine Chemotherapie alleine oder in Kombination mit einer konsolidierenden Bestrahlung erfolgen sollte, wird kontrovers diskutiert. Ziel dieser retrospektiven Analyse war es, Faktoren zu definieren, die die Therapieentscheidung erleichtern.
\end{abstract}

\begin{abstract}
n der Krebsdatenbank der American Cancer Society und des American College of Surgeons (National Cancer Data Base) wurden 59.255 Patienten mit DLBCL im Stadium I oder II identifiziert, die zwischen 1998 und 2012 eine Chemotherapie alleine oder in Kombination mit konsolidierender Bestrahlung erhalten hatten. $46 \%$ der Betroffenen hatten eine Erkrankung im Stadium II, $42 \%$ einen Extranodal-Befall. $58 \%$ waren über 60 Jahre alt. Nur $39 \%$ der Patienten erhielten eine kombinierte Therapie. Dieser Anteil nahm von 2000 (47\%) bis 2012 $(32 \%)$ signifikant ab $(\mathrm{p}<0,001)$, ebenso der Anteil der Patienten mit Bestrahlungsdosen über $36 \mathrm{~Gy}$ (von 62 auf $23 \%$ ). Dagegen wurde zunehmend häufiger
\end{abstract}

eine intensitätsmodulierte Radiotherapie durchgeführt (1998: $0 \%$; 2012: 24\%).

Die Wahrscheinlichkeit, eine kombinierte Therapie zu erhalten, war bei Vorliegen folgender Faktoren geringer: afroamerikanische Abstammung, mehr Komorbiditäten, keine Versicherung, schlechtere Ausbildung, Teilnahme an einem Forschungsprogramm, Alter über 60 Jahre, Stadium II, B-Symptome, Entfernung von mehr als 30 Meilen zur Therapieeinrichtung und eine kürzer zurückliegende Diagnose.

Die mediane Beobachtungsdauer betrug 60 Monate. Für alle Patienten lag die 5-Jahres-Gesamtüberlebensrate bei $79 \%$, für Patienten mit alleiniger Chemotherapie bei $75 \%$ und für Patienten mit kombinierter Therapie bei $82 \%$ ( $p<0,001)$. Die entsprechenden 10-Jahres-Überlebensraten betrugen $59 \%$, $55 \%$ und $64 \%(\mathrm{p}<0,001)$. Auch nach Berücksichtigung von Störfaktoren war die Kombinationstherapie mit einem besseren Gesamtüberleben assoziiert als die alleinige Chemotherapie (Hazard Ratio $0,66 ; \mathrm{p}<0,001)$.

Fazit: Heutzutage erfolgt bei Patienten mit DLBCL immer seltener eine Kombination aus Chemotherapie und Bestrahlung. Wird jedoch auf die Bestrahlung verzichtet, reduziert dies die Überlebenschancen der Patienten. Die Wahl der therapeutischen Strategie wird sowohl von klassischen prognostischen als auch von sozioökonomischen Faktoren beeinflusst.

Judith Neumaier

Vargo JA et al. Treatment Selection and Survival Outcomes in Early-Stage Diffuse Large B-Cell Lymphoma: Do We Still Need Consolidative Radiotherapy? J Clin Oncol. 2015;33(32):3710-7.

\section{Antikörper mit dualem Wirkmechanismus als Kombinationspartner beim multiplen Myelom}

\section{Die Einführung von Proteasominhibitoren und Immunmodulatoren verbes- serte das Therapieergebnis beim multiplen Myelom deutlich, die Rezidivrate ist aber weiterhin sehr hoch. Neue Ansätze sollen helfen, Therapieresistenzen zu überwinden und die langfristige Prognose zu verbessern.}

\footnotetext{
E in Standardtherapieregime für Pati— enten mit therapierefraktärem oder rezidiviertem multiplem Myelom (MM) ist die Kombination aus dem Immunmodulator Lenalidomid und Dexamethason (Len/Dex). Für erfolglos vorbehandelte Patienten kommen zunehmend Dreierkombinationen zum Einsatz, allerdings limitiert durch Toxizitäten. Erstrebenswert ist daher die Kombination aus traditionellen Substanzen und neuen, potenten Agenzien mit günstigerem Nebenwirkungsprofil.

Als vielversprechenden Kombinationspartner wählten Forscher den humanisierten monoklonalen Antikörper Elotuzumab, der gegen SLAMF7 („signa-
}

ling lymphocytic activation molecule F7") gerichtet ist. Dieses Glykoprotein ist auf Myelom- und natürlichen Killerzellen (NK) überexprimiert, nicht jedoch auf normalen Gewebezellen. Der erste Antikörper seiner Klasse aktiviert durch Bindung an SLAMF7 direkt NK und fördert zudem eine antikörperabhängige, zellvermittelte Zytotoxizität. In einer früheren Phase-II-Studie mit therapierefraktären oder rezidivierten MMPatienten verbesserte die Dreierkombination Elotuzumab/Len/Dex das progressionsfreie Überleben (PFS). Im Rahmen der randomisierten Phase-III-Studie ELOQUENT-2 verglichen die Forscher die Sicherheit und Wirksamkeit der
Dreierkombination $(\mathrm{n}=321)$ und der des Regimes Len/Dex $(n=325)$. Als Endpunkte legten sie PFS und Gesamtansprechen fest.

Einer geplanten Interimsanalyse zufolge betrug die PFS-Rate in der Elotuzumab-Gruppe nach einem Jahr $68 \%$ gegenüber $57 \%$ im Len/Dex-Arm, nach 2 Jahren $41 \%$ versus $27 \%$. Median lebten die Patienten der Verumgruppe 194 Monate progressionsfrei, in der Vergleichsgruppe 14,9 Monate. Die Vorteile der Dreierkombination spiegelten sich auch im Endpunkt Gesamtansprechrate wider: sie betrug 79 vs. $66 \%$. Insgesamt erwies sich der Antikörper als gut verträglich.

Fazit: Im Vergleich zur Behandlung mit Len/Dex reduzierte die Dreierkombination Elotuzumab/Len/Dex bei Patienten mit rezidiviertem oder refraktärem $\mathrm{MM}$ das Risiko für Progression oder Tod.

Wolfgang Zimmermann

Lonial S et al. Elotuzumab therapy for relapsed or refractory myeloma. New Eng J Med. 2015;373(7):621-31. 